\section{Methylphenidate-Induced Neuroleptic Malignant Syndrome: A Case Report}

Sir: Methylphenidate is a piperidine derivative, structurally related to amphetamines. It reaches peak blood concentration in 2 hours and has a short half-life of 1 to 2 hours. Its onset of action occurs shortly after dosing, and it can cause increased wakefulness, energy, alertness, and physical and mental performance. Its side effects include anorexia, weight loss, slow growth, insomnia, dysphoria, tics, and psychosis. There is a single case report of methylphenidate causing neuroleptic malignant syndrome (NMS) in a 1-year-old child with encephalomalacia. ${ }^{1}$ We report the first case of methylphenidate causing increased creatine kinase levels and possible neuroleptic malignant syndrome in a patient who had autistic disorder in the absence of any brain malformation.

Case report. A 10-year-old African American boy with autistic disorder (DSM-IV criteria) was admitted in 2002 to the child psychiatry inpatient unit as a crisis intervention measure to stabilize his numerous behavioral problems. The behavior of the patient was characterized by constant fidgetiness, restlessness, kicking, biting, and spitting. He also was throwing things and trying to run away. The patient was started on treatment with risperidone and clonazepam without success - he spit out his medications.

In view of the escalation of the patient's behavioral problems, intramuscular haloperidol and lorazepam were tried on an as-needed basis. After 2 injections each of haloperidol $(5 \mathrm{mg})$ and lorazepam $(2 \mathrm{mg})$ over 1 to 2 days, the patient had a dystonic reaction and was treated with diphenhydramine. However, after the patient received an additional 4 doses each of haloperidol (total of $20 \mathrm{mg}$ ) and lorazepam (total of $8 \mathrm{mg}$ ) by intramuscular injection over 2 days, he developed signs and symptoms suggestive of NMS, including fever, tachycardia, elevated white blood cell count, and creatine kinase (CK) levels elevated to over $8000 \mathrm{U} / \mathrm{L}$

The patient was transferred to the pediatric intensive care unit and treated appropriately by stopping haloperidol and starting intravenous hydration and sedation. The patient was transferred back to the psychiatric unit after his CK level, showing a downward trend, had decreased to $2000 \mathrm{U} / \mathrm{L}$. However, the behavioral problems in the form of aggression, spitting, and throwing things continued, and the patient was also constantly fidgety and restless.

At this stage, a trial of methylphenidate was considered with the hope of decreasing the patient's restlessness and fidgetiness. After he received the first and only dose of methylphenidate, $5 \mathrm{mg}$, he was noted to be relatively calm and less fidgety. However, the patient subsequently developed a fever, and his CK level increased from $690 \mathrm{U} / \mathrm{L}$ at the time of methylphenidate administration to $1033 \mathrm{U} / \mathrm{L} 5$ hours after methylphenidate administration. During this time, the patient was not agitated and not restrained and received no intramuscular injections that might explain the elevation of CK levels. One day after the administration of methylphenidate, the patient's fever subsided and his CK level dropped to $618 \mathrm{U} / \mathrm{L}$ and continued to drop further. No further doses of methylphenidate were administered.

Neuroleptic malignant syndrome is a potentially lifethreatening condition that presents with hyperthermia, mental status changes, oculogyric crisis, autonomic instability, and increased CK levels. Potential risk factors are previous episodes of NMS, psychomotor agitation, dehydration, rapid increase of neuroleptic medication, and parenteral routes of administration. Conventional and atypical antipsychotics have been implicated in its causation, as are any agents that possess dopaminergic antagonistic action.

In the present, we do not know if the patient would have developed all the signs and symptoms of NMS if he had continued to receive methylphenidate. However, he certainly had a significant CK level elevation that was related to methylphenidate. Since elevated CK levels are seen in about $95 \%$ of patients with NMS, ${ }^{2}$ it is likely that he might have developed a full-blown NMS.

It is possible that patients who have a propensity to develop NMS with antipsychotic medication are also likely to develop NMS with methylphenidate. Typically, NMS is caused by blockade of dopaminergic receptors. However, methylphenidate acts by inhibiting reuptake of dopamine, and therefore the mechanism by which it caused elevation of CK levels is not known.

Prior to this report, there has been only 1 case report describing neuroleptic malignant syndrome following methylphenidate administration, and that was in a 1-yearold child with encephalomalacia. ${ }^{1}$ This is the first case of NMS in a person without brain malformation. Methylphenidate should be used with caution in patients who have had an adverse reaction to antipsychotics. We believe that further experience is required before we can come to a definite conclusion that methylphenidate indeed can cause NMS and also before we can determine the mechanism by which it does so.

Drs. Basil, Mathews, and Budur, and report no financial or other relationships relevant to the subject of this letter.

\section{REFERENCES}

1. Ehara H, Maegaki Y, Takeshita K, Neuroleptic malignant syndrome and methylphenidate. Pediatr Neurol 1998;19:299-301

2. Mann SC, Caroff SN, Keck PE, et al. NMS and Related Conditions. 2nd ed. Washington, DC: American Psychiatric Press; 2003:1-44

Biju Basil, M.D., D.P.M. Maju Mathews, M.D., M.R.C.Psych. Department of Psychiatry Drexel University College of Medicine Philadelphia, Pennsylvania

Kumar Budur, M.D.

Department of Psychiatry Cleveland Clinic Cleveland, Ohio

\section{Aripiprazole Therapy for Nicotine Dependence}

Sir: Tobacco use poses a substantial health risk and increases morbidity and mortality, predominantly due to cardiovascular and respiratory pathophysiologic effects. The prevalence of nicotine dependence in the general population is about $24 \%{ }^{1}$ Nicotine dependence is relatively difficult to treat. Unassisted individual efforts at smoking cessation are associated with low success rates. With such 
efforts, as few as $3 \%$ to $5 \%$ of individuals remain abstinent at 1 year. $^{2}$

Bupropion is the only non-nicotine-based treatment that is approved for smoking cessation. We present a case report of aripiprazole therapy leading to smoking cessation.

Case report. Mr. A, a 21-year-old white man with a history of major depressive disorder, presented to the outpatient clinic for follow-up care. Approximately 1 month prior to this visit, he was hospitalized for worsening of depression with suicidal ideations. In addition, there was evidence of paranoid thoughts. At that time he also met DSM-IV criteria for alcohol abuse and nicotine dependence. He reported smoking 1 pack of cigarettes daily for the past 1 year. His Modified Fagerstrom Tolerance Questionnaire $^{3}$ score at admission was 7, indicative of severe nicotine dependence.

During his weeklong hospital stay, aripiprazole therapy was initiated. He was subsequently discharged on aripiprazole, 10 $\mathrm{mg}$ /day; trazodone, $50 \mathrm{mg}$ at bedtime; and acetaminophen, 650 mg 3 times daily. The patient was compliant with his outpatient treatment plan. While on aripiprazole therapy, the patient continued smoking for a few days. While on the above regimen of medications, he noticed a lack of "high" from the cigarettes as well as fewer cravings for nicotine use. Thus, he was able to quit smoking within a week of his discharge. His Modified Fagerstrom Tolerance Questionnaire score decreased to zero. He attributed these results to aripiprazole.

He continued to be nicotine abstinent for the next 2 weeks until he ran out of aripiprazole. While off aripiprazole therapy, he relapsed and went back to smoking. Approximately a week later, he resumed aripiprazole therapy and was simultaneously able to quit smoking again. During his outpatient visit, 5 weeks later, he did not meet criteria for nicotine dependence, which he again attributed to the aripiprazole. The patient was not drinking alcohol during his outpatient follow-up. However, he did not report any effect of aripiprazole on his desire for alcohol.

Current research in the neurobiology of substance abuse suggests that dopaminergic mechanisms are involved in motivation, reward, and reinforcement. It has been postulated that decreased dopamine function in addicted subjects results in decreased sensitivity to non-drug-related stimuli (including natural reinforcers) and disrupts frontal inhibition, both of which contribute to compulsive drug intake and impaired inhibitory control. ${ }^{4}$ Thus, enhancing dopamine function could theoretically help motivate addicted subjects in activities that provide alternative sources of reinforcement, counteract conditioned responses, enhance their ability to control their drive to take drugs, and interfere with their compulsive use. ${ }^{4}$

There have been several animal studies demonstrating dopamine release in response to nicotine administration. ${ }^{5-7}$ Also, there have been some human studies examining the effects of dopamine agonism and antagonism on smoking behavior. Haloperidol, a dopamine antagonist, has been shown to increase smoking in patients with schizophrenia ${ }^{8}$ and in nonpsychiatric patients. ${ }^{9,10}$ On the other hand, bromocriptine, a dopamine agonist, has been shown to significantly decrease smoking rate in a sample of nonpsychiatric patients. ${ }^{10}$ This hypothesis was further tested in a randomized double-blind fashion by observing the smoking behavior of 20 subjects after alternate administration of bromocriptine and haloperidol on separate occasions in the same subjects. A variety of cigarette smoking measures showed that subjects smoked more on haloperidol than on bromocriptine treatment. ${ }^{11}$ Bupropion, a drug approved for smoking cessation, is also thought to act by inhibiting neuronal norepinephrine and dopamine uptake, leading to enhanced synaptic dopamine concentration. ${ }^{12}$

Aripiprazole is a novel atypical antipsychotic drug with a unique mechanism of action: it inhibits central dopaminergic neuron activity by a partial agonistic effect on the presynaptic $\mathrm{D}_{2}$ dopamine autoreceptors and also acts as an antagonist at postsynaptic $\mathrm{D}_{2}$ dopamine receptors. ${ }^{13}$ Through this mechanism, aripiprazole exerts activity as a dopamine agonist in hypodopaminergic states while acting as a dopamine antagonist when dopaminergic activity is increased. ${ }^{14,15}$ Aripiprazole also acts as a partial agonist at $5-\mathrm{HT}_{1 \mathrm{~A}}$ serotonin receptors and as an antagonist at the $5-\mathrm{HT}_{2 \mathrm{~A}}$ serotonin receptors. ${ }^{13}$

Thinking in line with the current theory that hypodopaminergic states promote addictive behaviors, it is plausible that aripiprazole's dopamine-agonistic properties could be useful in the treatment of nicotine dependence. There is another case report suggesting that aripiprazole might decrease cravings and reduce alcohol use. ${ }^{16}$ In our case, the relationship between aripiprazole therapy and smoking cessation is further strengthened by the fact that the patient relapsed when he was off aripiprazole treatment. The reintroduction of aripiprazole decreased the reinforcing effects of nicotine and subsequently helped the patient stay abstinent.

This case suggests a cause-and-effect relationship between aripiprazole treatment and smoking cessation. It is also apparent that aripiprazole blocked the positive reinforcing properties of nicotine and decreased craving. In summary, the partial dopamine agonists such as aripiprazole are a promising class of drugs for treatment of addictive disorders. However, further research using double-blind placebo-controlled studies is needed to clarify this potential association. If this effect is replicated, aripiprazole would be a welcome addition to our treatment armamentarium for addictive disorders. Furthermore, if our observation is borne out in suitable studies, a patient's smoking status might be an additional factor to consider in selecting therapy for patients requiring an antipsychotic.

Dr. Bhatia has received honoraria from AstraZeneca, Bristol-Myers Squibb, Janssen, Pfizer, and Shire and has served on the speakers or advisory boards for AstraZeneca, Bristol-Myers Squibb, Janssen, Ortho-McNeil, and Shire. Dr. Ramaswamy reports no financial or other relationship relevant to the subject of this letter.

\section{REFERENCES}

1. Breslau N, Johnson EO, Hiripi E, et al. Nicotine dependence in the United States: prevalence, trends, and smoking persistence. Arch Gen Psychiatry 2001;58:810-816

2. Hughes JR. Tobacco withdrawal in self-quitters. J Consult Clin Psychol 1992;60:689-697

3. Heatherton TF, Kozlowski LT, Frecker RC, et al. The Fagerstrom Test for Nicotine Dependence: a revision of the Fagerstrom Tolerance Questionnaire. Br J Addict 1991;86:1119-1127

4. Volkow ND, Fowler JS, Wang GJ, et al. Dopamine in drug abuse and addiction: results from imaging studies and treatment implications. Mol Psychiatry 2004;9:557-569

5. Mifsud JC, Hernandez L, Hoebel BG. Nicotine infused into the nucleus accumbens increases synaptic dopamine as measured by in vivo microdialysis. Brain Res 1989;478:365-367

6. Nisell M, Nomikos GG, Svensson TH. Infusion of nicotine in the ventral tegmental area or the nucleus accumbens of the rat differentially affects accumbal dopamine release. Pharmacol Toxicol 1994; 75:348-352

7. Pontieri FE, Tanda G, Orzi F, et al. Effects of nicotine on the nucleus accumbens and similarity to those of addictive drugs. Nature 1996;382:255-257

8. McEvoy JP, Freudenreich O, Levin ED, et al. Haloperidol increases 
smoking in patients with schizophrenia. Psychopharmacology (Berl) 1995;119:124-126

9. Dawe S, Gerada C, Russell MA, et al. Nicotine intake in smokers increases following a single dose of haloperidol. Psychopharmacology (Berl) 1995;117:110-115

10. Caskey NH, Jarvik ME, Wirshing WC. The effects of dopaminergic D2 stimulation and blockade on smoking behavior. Exp Clin Psychopharmacol 1999;7:72-78

11. Caskey NH, Jarvik ME, Wirshing WC, et al. Modulating tobacco smoking rates by dopaminergic stimulation and blockade. Nicotine Tob Res 2002;4:259-266

12. Margolin A, Kosten TR, Avants SK, et al. A multicenter trial of bupropion for cocaine dependence in methadone-maintained patients. Drug Alcohol Depend 1995;40:125-131

13. DeLeon A, Patel NC, Crismon ML. Aripiprazole: a comprehensive review of its pharmacology, clinical efficacy, and tolerability.
Clin Ther 2004;26:649-666

14. Stahl SM. Dopamine system stabilizers, aripiprazole, and the next generation of antipsychotics, pt 1: "Goldilocks" actions at dopamine receptors [BRAinstorms]. J Clin Psychiatry 2001;62:841-842

15. Stahl SM. Dopamine system stabilizers, aripiprazole, and the next generation of antipsychotics, pt 2: illustrating their mechanism of action [Brainstorms]. J Clin Psychiatry 2001;62:923-924

16. Warsi M, Sattar SP, Bhatia SC, et al. Aripiprazole reduces alcohol use [letter]. Can J Psychiatry 2005;50:244

Sriram Ramaswamy, M.D.

Subhash C. Bhatia, M.D.

Department of Mental Health

Omaha Veterans Affairs Medical Center

Omaha, Nebraska 